\title{
GENERATORS AND RELATIONS OF REES MATRIX SEMIGROUPS
}

\author{
by H. AYIK* and N. RUŠKUC
}

(Received 25th August 1997)

\begin{abstract}
In this paper we consider finite generation and finite presentability of Rees matrix semigroups (with or without zero) over arbitrary semigroups. The main result states that a Rees matrix semigroup $\mathcal{M}[S ; I, J ; P]$ is finitely generated (respectively, finitely presented) if and only if $S$ is finitely generated (respectively, finitely presented), and the sets $I, J$ and $S \backslash U$ are finite, where $U$ is the ideal of $S$ generated by the entries of $P$.
\end{abstract}

1991 Mathematics subject classification: $20 \mathrm{M} 05$.

\section{Introduction and the main result}

Rees matrix semigroups were first introduced by Rees [15], although they were implicitly present in Suschkewitsch [19]. Since then, they have become one of the most important semigroup constructions, with numerous applications, especially to the structure theory of regular semigroups; for examples see $[8,9,10,11,12,14]$, and for a survey see [13]. In this paper we give necessary and sufficient conditions for a Rees matrix semigroup to be finitely generated or finitely presented.

Let $S$ be a semigroup, let $I$ and $J$ be two index sets, and let $P=\left(p_{j i}\right)_{j \in J, i \in I}$ be a $J \times I$ matrix with entries from $S$. The set

$$
I \times S \times J=\{(i, s, j) \mid i \in I, s \in S, j \in J\}
$$

with multiplication defined by

$$
(i, s, j)(k, t, l)=\left(i, s p_{j k} t, l\right)
$$

is a semigroup. This semigroup is called a Rees matrix semigroup, and is denoted by $\mathcal{M}[S ; I, J ; P]$.

If $S$ is a group, then $T$ is a completely simple semigroup, and, conversely, every completely simple semigroup can be obtained in this way; see [15] or [5]. There is a similar construction for completely 0 -simple semigroups; this is considered in Section 5 .

* Supported by Çukurova University, Adana, Turkey. 
Let $A$ be an alphabet. By $A^{+}$we denote the free semigroup on $A$, consisting of all non-empty words over $A$ under concatenation. We also let $A^{*}=A^{+} \cup\{\epsilon\}$, where $\epsilon$ is the empty word. A presentation is an ordered pair $\langle A \mid R\rangle$, where $R \subseteq A^{+} \times A^{+}$is a set of pairs of words. $S$ is said to be defined by $\langle A \mid R\rangle$ if $S \cong A^{+} / \rho$, where $\rho$ is the congruence generated by $R$. Thus we have a natural epimorphism $\pi_{s}: A^{+} \rightarrow S$ such that $R \subseteq \operatorname{ker}\left(\pi_{s}\right)$. For two words $w_{1}, w_{2} \in A^{+}$, we write $w_{1} \equiv w_{2}$ if they are identical words, and we write $w_{1}=w_{2}$ if they represent the same element of $S$ (i.e. if $\left.\pi_{s}\left(w_{1}\right)=\pi_{s}\left(w_{2}\right)\right)$. If $S$ can be defined by $\langle A \mid R\rangle$ with both $A$ and $R$ finite then $S$ is said to be finitely presented. Every finitely presented semigroup is finitely generated, but the converse is not true.

Now we give the main result of this paper.

Main Theorem. Let $S$ be a semigroup, let $I$ and $J$ be index sets, let $P=\left(p_{j i}\right)_{j \in J_{i} \in I}$ be $a J \times I$ matrix with entries from $S$, and let $U$ be the ideal of $S$ generated by the set $\left\{p_{j i} \mid j \in J, i \in I\right\}$ of all entries of $P$. Then the Rees matrix semigroup $\mathcal{M}[S ; I, J ; P]$ is finitely generated (respectively, finitely presented) if and only if the following three conditions are satisfied:

(i) both $I$ and $J$ are finite;

(ii) $S$ is finitely generated (respectively, finitely presented); and

(iii) the set $S \backslash U$ is finite.

Proof. The result for finite generation follows from Propositions 2.1 and 2.2 , and Corollary 2.4. The result for finite presentability then follows from Corollaries 3.5 and 4.5 .

\section{Generators}

The purpose of this section is twofold. In it we prove the part of the Main Theorem concerning finite generation of the Rees matrix semigroup $T=\mathcal{M}[S ; I, J ; P]$. In the process we also construct certain natural generating sets for $S$ and $T$, thus preparing the ground for the considerations in Sections 3 and 4.

Proposition 2.1. Let $T=\mathcal{M}[S ; I, J ; P]$ be a Rees matrix semigroup, and let $U$ be the ideal of $S$ generated by the entries of $P$. If $T$ is finitely generated then $I, J$ and $S \backslash U$ are finite sets.

Proof. Observe that, for any $(i, s, j),(k, t, l) \in T$, we have $(i, s, j)(k, t, l)=\left(i, s p_{j k} t, l\right)$ and $s p_{j k} t \in U$. Therefore every element of the set $I \times S \backslash U \times J$ is indecomposable (i.e. not equal to the product of two elements from $T$ ), and hence belongs to every generating set of $T$. Therefore, if $T$ is finitely generated, each of $I, J$ and $S \backslash U$ must be finite, as required. 
Next we describe a generating set for $S$, given a generating set for $T$.

Proposition 2.2. If $X$ is a generating set for a Rees matrix semigroup $T=$ $\mathcal{M}[S ; I, J ; P]$, then the set

$$
Y=\{s \in S \mid(i, s, j) \in X \text { for some } i \in I, j \in J\} \cup\left\{p_{j i} \mid j \in J, i \in I\right\}
$$

generates $S$.

Proof. Let $s \in S$ be arbitrary. By taking arbitrary $i \in I, j \in J$, and decomposing

$$
(i, s, j)=\left(i_{1}, s_{1}, j_{1}\right) \ldots\left(i_{m}, s_{m}, j_{m}\right)=\left(i_{1}, s_{1} p_{j_{1} i_{2}} s_{2} \ldots p_{j_{m-1} i_{m}} s_{m}, j_{m}\right)
$$

into a product of generators $\left(i_{1}, s_{1}, j_{1}\right), \ldots,\left(i_{m}, s_{m}, j_{m}\right) \in X$, we conclude that $s=$ $s_{1} p_{j_{1} i_{2}} s_{2} \cdots p_{j_{m-1} i_{m}} s_{m} \in\langle Y\rangle$, as required.

In the next result, we construct a natural generating set for $T$, assuming that $S$ is a monoid.

Proposition 2.3. Let $S$ be a monoid and let $T=\mathcal{M}[S ; I, J ; P]$ be a Rees matrix semigroup. Denote by $U$ the ideal of $S$ generated by the entries of $P$, and let $Z$ be a set generating $U$ as a semigroup. Write an arbitrary element $z \in Z$ as $s(z) p_{j(z) i(z)} s^{\prime}(z)$, with $s(z)$, $s^{\prime}(z) \in S, j(z) \in J$ and $i(z) \in I$, and let $H=\left\{s(z), s^{\prime}(z) \mid z \in Z\right\} \cup\{1\}$. Then the set $X=I \times\left(H^{2} \cup S \backslash U\right) \times J$ generates $T$.

Proof. Take an arbitrary element $(i, s, j) \in T$. If $s \notin U$, then $(i, s, j) \in X$. Assume that $s \in U$, say $s=z_{1} \cdots z_{m}$, where $z_{1}, \ldots, z_{m} \in Z$. Then we have

$$
\begin{aligned}
(i, s, j) & =\left(i, z_{1} \cdots z_{m}, j\right)=\left(i, s\left(z_{1}\right) p_{j\left(z_{1}\right) i\left(z_{1}\right)} s^{\prime}\left(z_{1}\right) \cdots s\left(z_{m}\right) p_{j\left(z_{m}\right)\left(z_{m}\right)} s^{\prime}\left(z_{m}\right), j\right) \\
& =\left(i, s\left(z_{1}\right), j\left(z_{1}\right)\right)\left(i\left(z_{1}\right), s^{\prime}\left(z_{1}\right) s\left(z_{2}\right), j\left(z_{2}\right)\right) \cdots\left(i\left(z_{m}\right), s^{\prime}\left(z_{m}\right), j\right) \in\langle X\rangle,
\end{aligned}
$$

completing the proof.

Remark. If $S$ is not a monoid, one can still construct a generating set for $T$ along the same lines, replacing $H^{2}$ by the set $\left\{s(z), s^{\prime}(z) \mid z \in Z\right\} \cup\left\{s^{\prime}(z) s(z) \mid z \in Z\right\}$. However, it is the generating set given in Proposition 2.3 which will prove useful in Section 4. Alternatively, when $S$ is not a monoid one may note that $\mathcal{M}[S ; I, J ; P]$ is a subsemigroup of $\mathcal{M}\left[S^{1} ; I, J ; P\right]$ (where, as usual, $S^{1}$ denotes the monoid obtained from $S$ by adjoining an identity element if necessary), and then use the methods from [7] or [1, $2,18]$ to obtain a generating set for $\mathcal{M}[S ; I, J ; P]$. This idea is used in the following:

Corollary 2.4. In the notation of the Main Theorem, if $I, J$ and $S \backslash U$ are all finite and $S$ is finitely generated, then the Rees matrix semigroup $\mathcal{M}[S ; I, J ; P]$ is finitely generated. 
Proof. Since $S$ is finitely generated and $S \backslash U$ is finite, it follows by [18, Theorem 1.1] that $U$ is finitely generated as a semigroup. Therefore, by Proposition 2.3, $T^{\prime}=\mathcal{M}\left[S^{1} ; I, J ; P\right]$ is also finitely generated. Finally, note that $T^{\prime} \backslash T \subseteq I \times\{1\} \times J$ is finite, so that $T$ is finitely generated by [18, Theorem 1.1].

\section{Presentations (1)}

In this section we construct a presentation for a semigroup $S$, starting from a presentation for a Rees matrix semigroup $T=\mathcal{M}[S ; I, J ; P]$. This presentation for $S$ is finite whenever the starting presentation for $T$ is finite, and so we have a proof of the direct part of the Main Theorem concerning finite presentability.

Let $H=\{(i(k), s(k), j(k)) \mid k \in K\}$ be a generating set for $T=\mathcal{M}[S ; I, J ; P]$. If we define $Y=\{s(k) \mid k \in K\}$, then it is clear that the set $X=I \times Y \times J$ contains $H$, and so generates $T$. Moreover, by Proposition 2.1, $X$ is finite if and only if $H$ is finite.

Take an alphabet

$$
A=\{a(i, y, j) \mid i \in I, y \in Y, j \in J\}
$$

in one-one correspondence with $X$. Let $\langle A \mid R\rangle$ be a presentation for $T$ in terms of $X$, and let

$$
\pi_{r}: A^{+} \rightarrow T, \quad a(i, y, j) \mapsto(i, y, j),
$$

be the natural projection. By Proposition 2.2, the set

$$
Y \cup\left\{p_{j i} \mid j \in J, i \in I\right\}
$$

generates $S$. Take a new alphabet

$$
C=\{c(y) \mid y \in Y\} \cup\{d(j, i) \mid j \in J, i \in I\},
$$

and let

$$
\pi_{s}: C^{+} \rightarrow S, \quad c(y) \mapsto y, \quad d(j, i) \mapsto p_{j i}
$$

be the natural projection.

Next we define a mapping $\psi: A^{+} \rightarrow C^{+}$by

$$
\psi\left(a\left(i_{1}, y_{1}, j_{1}\right) \cdots a\left(i_{m}, y_{m}, j_{m}\right)\right)=c\left(y_{1}\right) d\left(j_{1}, i_{2}\right) c\left(y_{2}\right) \cdots d\left(j_{m-1}, i_{m}\right) c\left(y_{m}\right),
$$

where $i_{1}, \ldots, i_{m} \in I, y_{1}, \ldots, y_{m} \in Y$, and $j_{1}, \ldots, j_{m} \in J$. (Intuitively, $\psi$ rewrites a word $w \in A^{+}$into a word from $C^{+}$which represents the middle component of the element $\left.\pi_{T}(w) \in T.\right)$

For a word $w \equiv a\left(i_{1}, y_{1}, j_{1}\right) \cdots a\left(i_{m}, y_{m}, j_{m}\right) \in A^{+}$define $\lambda(w)=i_{1}$ and $\rho(w)=j_{m}$. With 
this notation, the above definition of $\psi$ has the following immediate consequence

$$
\psi\left(w_{1} w_{2}\right) \equiv \psi\left(w_{1}\right) d\left(\rho\left(w_{1}\right), \lambda\left(w_{2}\right)\right) \psi\left(w_{2}\right)
$$

for all $w_{1}, w_{2} \in A^{+}$.

If we let $W=\operatorname{im}(\psi)$, then we have the following:

Lemma 3.1. For all $y, y^{\prime} \in Y, i \in I, j \in J$, there exist words $\zeta\left(y, y^{\prime}\right), \eta(j, i) \in W$ such that the relations

hold in $S$.

$$
\begin{gathered}
c(y) c\left(y^{\prime}\right)=\zeta\left(y, y^{\prime}\right), \\
d(j, i)=\eta(j, i)
\end{gathered}
$$

Proof. Let $w \in C^{+}, \quad i_{0} \in I, \quad j_{0} \in J$ be arbitrary, and consider the element $\left(i_{0}, \pi_{s}(w), j_{0}\right) \in T$. If we write

$$
\left(i_{0}, \pi_{s}(w), j_{0}\right)=\left(i_{1}, y_{1}, j_{1}\right) \cdots\left(i_{m}, y_{m}, j_{m}\right)
$$

a product of generators from $X$, we conclude that

$$
\pi_{s}(w)=y_{1} p_{j_{1} i_{2}} y_{2} \cdots p_{j_{m-1} i_{m}} y_{m}
$$

For $w^{\prime} \equiv c\left(y_{1}\right) d\left(j_{1}, i_{2}\right) c\left(y_{2}\right) \cdots d\left(j_{m-1}, i_{m}\right) c\left(y_{m}\right)$, we now have $w^{\prime} \in W$, and the relation $w=w^{\prime}$ holds in $S$. By putting in the above argument $w \equiv c(y) c\left(y^{\prime}\right)$ and $w \equiv d(j, i)$ respectively, we complete the proof.

For the remainder of this section, we consider the words $\zeta\left(y, y^{\prime}\right), \eta(j, i)$ to be fixed in accordance with Lemma 3.1.

Lemma 3.2. There exists a mapping $\sigma: C^{+} \rightarrow W$ such that the relation $w=\sigma(w)$ is a consequence of the relations (5) and (6).

Proof. If $w \in W$, then define $\sigma(w)=w$. If $w \notin W$ then first apply (6) to obtain a word $c\left(y_{1}\right) w_{1} c\left(y_{2}\right)$ containing no subword of the form $d\left(j_{1}, i_{2}\right) d\left(j_{2}, i_{2}\right)$, and then systematically apply (5) to eliminate all the subwords of the form $c(y) c\left(y^{\prime}\right)$.

Intuitively, $\sigma$ rewrites an arbitrary word from $C^{+}$into a corresponding word in the image of $\psi$. We now use $\sigma$ to define a mapping $\phi: C^{+} \rightarrow A^{+}$, which will act as a kind of inverse to $\psi$, as follows:

$$
\phi(w)=a\left(i_{0}, y_{1}, j_{1}\right) a\left(i_{2}, y_{2}, j_{2}\right) \cdots a\left(i_{m}, y_{m}, j_{0}\right)
$$


where $i_{0} \in I$ and $j_{0} \in J$ are fixed, and where

$$
\sigma(w) \equiv c\left(y_{1}\right) d\left(j_{1}, i_{2}\right) c\left(y_{2}\right) \cdots d\left(j_{m-1}, i_{m}\right) c\left(y_{m}\right)
$$

Finally, we let $\mu: T \rightarrow S,(i, s, j) \mapsto s$ be the second projection. In the following lemma, we establish certain connections between $\pi_{s}, \pi_{T}, \phi, \psi$ and $\mu$.

Lemma 3.3. (i) For any word $w \in C^{+}$, we have $\mu \pi_{T} \phi(w)=\pi_{s}(w)$.

(ii) For any word $w \in A^{+}$, we have $\mu \pi_{T}(w)=\pi_{s} \psi(w)$.

Proof. (i) If $\sigma(w) \equiv c\left(y_{1}\right) d\left(j_{1}, i_{2}\right) c\left(y_{2}\right) \cdots d\left(j_{m-1}, i_{m}\right) c\left(y_{m}\right)$, then

$$
\begin{array}{rlrl}
\mu \pi_{T} \phi(w) & =\mu \pi_{T}\left(a\left(i_{0}, y_{1}, j_{1}\right) a\left(i_{2}, y_{2}, j_{2}\right) \cdots a\left(i_{m}, y_{m}, j_{0}\right)\right) & & (\text { by }(7)) \\
& =\mu\left(\left(i_{0}, y_{1}, j_{1}\right)\left(i_{2}, y_{2}, j_{2}\right) \cdots\left(i_{m}, y_{m}, j_{0}\right)\right) & & (\text { by }(1)) \\
& =\mu\left(\left(i_{0}, y_{1} p_{j_{1} i_{2}} y_{2} \cdots p_{j_{m-1} i_{m}} y_{m}, j_{0}\right)\right) & \\
& =y_{1} p_{j_{1} i_{2}} y_{2} \cdots p_{j_{m-1} i_{m}} y_{m} & \\
& =\pi_{s} \sigma(w) & & \text { (by (2)) } \\
& =\pi_{s}(w) . & & \text { (Lemma }
\end{array}
$$

The proof of (ii) is similar.

Now we can state and prove the main result of this section.

Theorem 3.4. Let $T=\mathcal{M}[S ; I, J ; P]$ be a Rees matrix semigroup and let $\langle A \mid R\rangle$ be a presentation for $T$ in terms of a generating set of the form $I \times Y \times J$, with $Y \subseteq S$. With the above notation, $S$ is defined by the presentation

$$
\begin{array}{cl}
\langle C| \psi(u)=\psi(v) & ((u=v) \in R) \\
c(y) c\left(y^{\prime}\right)=\zeta\left(y, y^{\prime}\right) & \left(y, y^{\prime} \in Y\right) \\
d(j, i)=\eta(j, i) & (j \in J, i \in I)\rangle
\end{array}
$$

in terms of the generating set $Y \cup\left\{p_{j i} \mid j \in J, i \in I\right\}$.

Proof. Since the relation $u=v$ holds in $T$, it follows that $\pi_{T}(u)=\pi_{T}(v)$, and so, by Lemma 3.3 (ii), we have

$$
\pi_{S} \psi(u)=\mu \pi_{T}(u)=\mu \pi_{T}(v)=\pi_{S} \psi(v) .
$$

Thus, all the relations (9) hold in $S$. That all the relations (10) and (11) hold in $S$ follows from Lemma 3.1.

To complete the proof of the theorem, we show that an arbitrary relation $w_{1}=w_{2}$ 
$\left(w_{1}, w_{2} \in C^{+}\right)$which holds in $S$ is a consequence of (9), (10) and (11). We do this in three steps.

Step 1: The relation $\phi\left(w_{1}\right)=\phi\left(w_{2}\right)$ holds in T. Indeed, by Lemma 3.3 (i), (1) and (7), we have:

$$
\begin{aligned}
\pi_{T} \phi\left(w_{1}\right) & =\left(i_{0}, \mu \pi_{T} \phi\left(w_{1}\right), j_{0}\right)=\left(i_{0}, \pi_{s}\left(w_{1}\right), j_{0}\right)=\left(i_{0}, \pi_{S}\left(w_{2}\right), j_{0}\right) \\
& =\left(i_{0}, \mu \pi_{T} \phi\left(w_{2}\right), j_{0}\right)=\pi_{T} \phi\left(w_{2}\right) .
\end{aligned}
$$

Step 2: The relation $\psi \phi\left(w_{1}\right)=\psi \phi\left(w_{2}\right)$ is a consequence of (9). From Step 1, we know that $\phi\left(w_{2}\right)$ can be obtained from $\phi\left(w_{1}\right)$ by applying relations from $R$. Without loss of generality, we can assume that $\phi\left(w_{2}\right)$ can be obtained from $\phi\left(w_{1}\right)$ by one application of one relation $(u=v) \in R$, i.e. that

$$
\phi\left(w_{1}\right) \equiv \alpha u \beta \quad \text { and } \quad \phi\left(w_{2}\right) \equiv \alpha v \beta,
$$

for some $\alpha, \beta \in A^{*}$. If both $\alpha$ and $\beta$ are non-empty, then we have

$$
\begin{array}{rlrl}
\psi \phi\left(w_{1}\right) & \equiv \psi(\alpha u \beta) & \\
& \equiv \psi(\alpha) d(\rho(\alpha), \lambda(u)) \psi(u) d(\rho(u), \lambda(\beta)) \psi(\beta) & & (\text { by }(4)) \\
& =\psi(\alpha) d(\rho(\alpha), \lambda(u)) \psi(v) d(\rho(u), \lambda(\beta)) \psi(\beta) & & (\text { relation }(9)) \\
& \equiv \psi(\alpha) d(\rho(\alpha), \lambda(v)) \psi(v) d(\rho(v), \lambda(\beta)) \psi(\beta) & & (\text { since } u=v \text { in } T) \\
& \equiv \psi \phi\left(w_{1}\right) .
\end{array}
$$

The case where at least one of $\alpha$ or $\beta$ is empty is treated similarly.

Step 3: The relations $\psi \phi\left(w_{k}\right)=w_{k}, k=1,2$, are consequences of (10) and (11). Indeed, by (3), (7), (8) and Lemma 3.2, we have

$$
\psi \phi\left(w_{k}\right) \equiv \sigma\left(w_{k}\right)=w_{k} \quad(k=1,2),
$$

a consequence of (10) and (11).

The proof of the theorem is now complete.

Corollary 3.5. If $T=\mathcal{M}[S ; I, J ; P]$ is finitely presented, then so is $S$.

Proof. As explained at the beginning of this section, if $T$ is finitely generated, then it has a finite generating set of the form $I \times Y \times J$. Moreover, if $T$ is finitely presented, it can be defined by a finite presentation $(A \mid R)$ in terms of this generating set. An application of the previous theorem to $\langle A \mid R\rangle$ yields a finite presentation for $S$. 


\section{Presentations (2)}

Now we find a presentation for a Rees matrix semigroup $T=\mathcal{M}[S ; I, J ; P]$, given a presentation for the ideal $U$ of $S$ generated by the entries of $P$. We do this in the case where $S$ is a monoid. Then we use the main result of [18] to extend this presentation to the case where $S$ is an arbitrary semigroup, and then to complete the proof of the Main Theorem. The argument in this section is in outline similar to that of Section 3, and is based on the idea of rewriting the given relations. However, the technical details are different and slightly more complicated.

So let $S$ be a monoid, let $T=\mathcal{M}[S ; I, J ; P]$ be a Rees matrix semigroup, and let $U$ be the ideal generated by the set $\left\{p_{j i} \mid j \in J, i \in I\right\}$ of all entries of $P$. Let $Z \subseteq U$ be any set generating $U$ as a semigroup. As in Proposition 2.3, write an arbitrary element $z \in Z$ as

$$
z=s(z) p_{j(z) i(z)} s^{\prime}(z)
$$

with $s(z), s^{\prime}(z) \in S, i(z) \in I, j(z) \in J$, and let

$$
H=\left\{s(z), s^{\prime}(z) \mid z \in Z\right\} \cup\{1\} .
$$

Then, clearly, the set

$$
Y=\left\{h p_{j i} h^{\prime} \mid h, h^{\prime} \in H, j \in J, i \in I\right\}
$$

contains $Z$, and hence generates $U$ as a semigroup. Moreover, $Y$ is finite, provided that $Z, I$ and $J$ are all finite.

Now let

$$
C=\left\{c\left(h, j, i, h^{\prime}\right) \mid h, h^{\prime} \in H, j \in J, i \in I\right\}
$$

be a new alphabet representing elements of $Y$, and let $\langle C \mid R\rangle$ be a presentation for $U$. For technical reasons, we also introduce an alphabet

$$
D=\{d(s) \mid s \in S \backslash U\}
$$

representing elements of $S \backslash U$. It is obvious that the set $Y \cup S \backslash U$ generates $S$, and so the natural homomorphism

$$
\pi_{s}:(C \cup D)^{+} \rightarrow S, \quad c\left(h, j, i, h^{\prime}\right) \mapsto h p_{j i} h^{\prime}, \quad d(s) \mapsto s
$$

is onto. By Proposition 2.3, the set

$$
X=I \times\left(H^{2} \cup S \backslash U\right) \times J
$$

generates $T$. Let 


$$
\begin{gathered}
A=\left\{a\left(i, h^{\prime}, h, j\right) \mid i \in I, h^{\prime}, h \in H, j \in J\right\} \quad \text { and } \\
B=\{b(i, s, j) \mid i \in I, s \in S \backslash U, j \in J\}
\end{gathered}
$$

be two alphabets, and let

$$
\pi_{T}:(A \cup B)^{+} \rightarrow T, \quad a\left(i, h^{\prime}, h, j\right) \mapsto\left(i, h^{\prime} h, j\right), \quad b(i, s, j) \mapsto(i, s, j)
$$

be the natural projection.

Next, we define a mapping $\phi: I \times H \times C^{+} \times H \times J \rightarrow A^{+}$by

$$
\phi\left(i, h^{\prime}, w, h, j\right)=a\left(i, h^{\prime}, h_{1}, j_{1}\right) a\left(i_{1}, h_{1}^{\prime}, h_{2}, j_{2}\right) \cdots a\left(i_{m}, h_{m}^{\prime}, h, j\right)
$$

where $w \equiv c\left(h_{1}, j_{1}, i_{1}, h_{1}^{\prime}\right) \cdots c\left(h_{m}, j_{m}, i_{m}, h_{m}^{\prime}\right) \in C^{+}$. Intuitively, $\phi\left(i, h^{\prime}, w, h, j\right)$ is a word in $A^{+}$representing the element $\left(i, h^{\prime} \pi_{s}(w) h, j\right) \in T$. Immediately from the above definition of $\phi$, it follows that

$$
\phi\left(i, h^{\prime}, w_{1} c\left(h_{1}, j_{1}, i_{1}, h_{1}^{\prime}\right) w_{2}, h, j\right) \equiv \phi\left(i, h^{\prime}, w_{1}, h_{1}, j_{1}\right) \phi\left(i_{1}, h_{1}^{\prime}, w_{2}, \boldsymbol{h}, j\right)
$$

for all $i, i_{1} \in I, j, j_{1} \in J, h, h^{\prime}, h_{1}, h_{1}^{\prime} \in H$ and all $w_{1}, w_{2} \in C^{*}$, where we introduce the convention that $\phi\left(i, h^{\prime}, \epsilon, h, j\right) \equiv a\left(i, h^{\prime}, h, j\right)$.

We also need a mapping $(A \cup B)^{+} \rightarrow(C \cup D)^{+}$, which would rewrite a word $w \in(A \cup B)^{+}$into a word representing the middle component of $\pi_{T}(w)$. To this end, we let

$$
W=\left\{\phi(i, 1, w, 1, j) \mid i \in I, w \in A^{+}, j \in J\right\},
$$

and then establish certain relations allowing us to transform words from $(A \cup B)^{+}$into words from $W$.

Lemma 4.1. For arbitrary $i, i^{\prime}, i^{\prime \prime} \in I, j, j^{\prime}, j^{\prime \prime} \in J, h, h^{\prime} \in H$ and $s^{\prime}, s^{\prime \prime} \in S \backslash U$, there exist words $\zeta\left(i, h^{\prime}, h, j\right) \in W \cup B, \eta\left(i^{\prime}, i^{\prime \prime}, j^{\prime}, j^{\prime \prime}, s^{\prime}, s^{\prime \prime}\right), \theta\left(i, i^{\prime}, j, j^{\prime}, h, h^{\prime}, s^{\prime}\right), \lambda\left(i, i^{\prime}, j, j^{\prime}, h, h^{\prime}, s^{\prime}\right) \in W$ such that the relations

$$
\begin{aligned}
& a\left(i, h^{\prime}, h, j\right)=\zeta\left(i, h^{\prime}, h, j\right), \\
& b\left(i^{\prime}, s^{\prime}, j^{\prime}\right) b\left(i^{\prime \prime}, s^{\prime \prime}, j^{\prime \prime}\right)=\eta\left(i^{\prime}, i^{\prime \prime}, j^{\prime}, j^{\prime \prime}, s^{\prime}, s^{\prime \prime}\right), \\
& b\left(i^{\prime}, s^{\prime}, j^{\prime}\right) a\left(i, h^{\prime}, h, j\right)=\theta\left(i, i^{\prime}, j, j^{\prime}, h, h^{\prime}, s^{\prime}\right), \\
& a\left(i, h^{\prime}, h, j\right) b\left(i^{\prime}, s^{\prime}, j^{\prime}\right)=\lambda\left(i, i^{\prime}, j, j^{\prime}, h, h^{\prime}, s^{\prime}\right)
\end{aligned}
$$

hold in $T$.

Proof. Let $w \in(A \cup B)^{+}$be arbitrary, and write $\pi_{T}(w)=\left(i_{0}, s, j_{0}\right)$. If $s \in S \backslash U$ then define $w^{\prime} \equiv b\left(i_{0}, s, j_{0}\right)$. Otherwise, if $s \in U$, then we can write 


$$
s=h_{1} p_{j_{1} i_{1}} h_{1}^{\prime} \cdots h_{m} p_{j_{m_{m}}} h_{m}^{\prime}
$$

a product of generators from $Y$, and then define

$$
w^{\prime} \equiv a\left(i_{0}, 1, h_{1}, j_{1}\right) a\left(i_{1}, h_{1}^{\prime}, h_{2}, j_{2}\right) \cdots a\left(i_{m}, h_{m}^{\prime}, 1, j_{0}\right) \in W
$$

With this choice we have $w^{\prime} \in W \cup B$, and the relation $w=w^{\prime}$ holds in $T$. The proof of the lemma is completed by letting $w \equiv a\left(i, h^{\prime}, h, j\right), w \equiv b\left(i^{\prime}, s^{\prime}, j^{\prime}\right) b\left(i^{\prime \prime}, s^{\prime \prime}, j^{\prime \prime}\right)$, $w \equiv b\left(i^{\prime}, s^{\prime}, j^{\prime}\right) a\left(i, h^{\prime}, h, j\right)$ and $w \equiv a\left(i, h^{\prime}, h, j\right) b\left(i^{\prime}, s^{\prime}, j^{\prime}\right)$ respectively, and noting that in the last three cases we cannot have $w^{\prime} \in B$.

For the remainder of this section, we consider the words $\zeta, \eta, \theta$ and $\lambda$ to be fixed.

Lemma 4.2. There exists a mapping $\sigma:(A \cup B)^{+} \rightarrow W \cup B$ such that the relation $w=\sigma(w)$ is a consequence of relations (16)-(19) for every word $w \in(A \cup B)^{+}$.

Proof. Let $w \in(A \cup B)^{+}$. First replace each $a\left(i, h^{\prime}, h, j\right)$ in $w$ by the corresponding $\zeta\left(i, h^{\prime}, h, j\right)$. If the resulting word is $b\left(i^{\prime}, s^{\prime}, j^{\prime}\right)$ define $\sigma(w) \equiv b\left(i^{\prime}, s^{\prime}, j^{\prime}\right)$. Otherwise, use (17), (18) and (19) to eliminate systematically all symbols $b\left(i^{\prime}, s^{\prime}, j^{\prime}\right)$, and define $\sigma(w)$ to be the resulting word.

Now we define the required mapping $\psi:(A \cup B)^{+} \rightarrow(C \cup D)^{+}$as follows

$$
\psi(w)=\left\{\begin{aligned}
d(s) \quad \text { if } \quad \sigma(w) \equiv b(i, s, j) \\
c\left(h_{1}, j_{1}, i_{1}, h_{1}^{\prime}\right) \cdots c\left(h_{m}, j_{m}, i_{m}, h_{m}^{\prime}\right) \\
\quad \text { if } \sigma(w) \equiv a\left(i, 1, h_{1}, j_{1}\right) a\left(i_{1}, h_{1}^{\prime}, h_{2}, j_{2}\right) \cdots a\left(i_{m}, h_{m}^{\prime}, 1, j\right)
\end{aligned}\right.
$$

We also let, as before, $\mu: T \rightarrow S,(i, s, j) \mapsto s$, be the second projection.

Lemma 4.3. (i) For all $w \in(A \cup B)^{+}$, we have $\pi_{s} \psi(w)=\mu \pi_{T}(w)$.

(ii) For all $w \in C^{+}, i \in I, j \in J, h, h^{\prime} \in H$, we have $\mu \pi_{T} \phi\left(i, h^{\prime}, w, h, j\right)=h^{\prime} \pi_{s}(w) h$.

Proof. (i) If $\sigma(w) \equiv b(i, s, j)$ then

$$
\pi_{s} \psi(w)=\pi_{s}(d(s))=s=\mu((i, s, j))=\mu \pi_{T}(b(i, s, j))=\mu \pi_{T} \sigma(w)=\mu \pi_{T}(w)
$$

by (20), (12), (13), and Lemmas 4.1 and 4.2 , while if

$$
\sigma(w) \equiv a\left(i, 1, h_{1}, j_{1}\right) a\left(i_{1}, h_{1}^{\prime}, h_{2}, j_{2}\right) \cdots a\left(i_{m}, h_{m}^{\prime}, 1, j\right) \in W,
$$

then 


$$
\begin{array}{rlrl}
\pi_{s} \psi(w) & =\pi_{s}\left(c\left(h_{1}, j_{1}, i_{1}, h_{1}^{\prime}\right) \cdots c\left(h_{m}, j_{m}, i_{m}, h_{m}^{\prime}\right)\right) & & \text { (by (20)) } \\
& =h_{1} p_{j_{1} i_{1}} h_{1}^{\prime} \cdots h_{m} p_{j_{m} i_{m}} h_{m}^{\prime} & & \text { by (12)) } \\
& =\mu\left(\left(i, h_{1} p_{j_{1} i_{1}} h_{1}^{\prime} \cdots h_{m} p_{j_{m} i_{m}} h_{m}^{\prime}, j\right)\right) & \\
& =\mu \pi_{T}\left(a\left(i, 1, h_{1}, j_{1}\right) a\left(i_{1}, h_{1}^{\prime}, h_{2}, j_{2}\right) \cdots a\left(i_{m}, h_{m}^{\prime}, 1, j\right)\right) & & \text { (by (13)) } \\
& =\mu \pi_{T}(w) . & & \text { Lemmas }
\end{array}
$$

(ii) is proved similarly.

Now we state and prove the main result of this section.

Theorem 4.4. Let $S$ be a monoid, let $T=\mathcal{M}[S ; I, J ; P]$ be a Rees matrix semigroup, and let $U$ be the ideal of $S$ generated by $\left\{p_{j i} \mid j \in J, i \in I\right\}$. If $\langle C \mid R\rangle$ is a presentation for $U$ in terms of a generating set $Y=\left\{h p_{j i} h^{\prime} \mid h, h^{\prime} \in H, j \in J, i \in I\right\}$ with $1 \in H \subseteq S$, then, with the above notation, the presentation with generators $A \cup B$ and relations

$$
\begin{aligned}
& \phi\left(i, h^{\prime}, u, h, j\right)=\phi\left(i, h^{\prime}, v, h, j\right), \\
& a\left(i, h^{\prime}, h, j\right)=\zeta\left(i, h^{\prime}, h, j\right), \\
& b\left(i^{\prime}, s^{\prime}, j^{\prime}\right) b\left(i^{\prime \prime}, s^{\prime \prime}, j^{\prime \prime}\right)=\eta\left(i^{\prime}, i^{\prime \prime}, j^{\prime}, j^{\prime \prime}, s^{\prime}, s^{\prime \prime}\right), \\
& b\left(i^{\prime}, s^{\prime}, j^{\prime}\right) a\left(i, h^{\prime}, h, j\right)=\theta\left(i, i^{\prime}, j, j^{\prime}, h, h^{\prime}, s^{\prime}\right), \\
& a\left(i, h^{\prime}, h, j\right) b\left(i^{\prime}, s^{\prime}, j^{\prime}\right)=\lambda\left(i, i^{\prime}, j, j^{\prime}, h, h^{\prime}, s^{\prime}\right),
\end{aligned}
$$

where $(u=v) \in R, i, i^{\prime}, i^{\prime \prime} \in I, j, j^{\prime}, j^{\prime \prime} \in J, h, h^{\prime} \in H, s^{\prime}, s^{\prime \prime} \in S \backslash U$, defines $T$ in terms of the generating set $X=I \times\left(H^{2} \cup S \backslash U\right) \times J$.

Proof. Note that by (13), (14) and Lemma 4.3 (ii), we have

$$
\begin{aligned}
\pi_{T} \phi\left(i, h^{\prime}, u, h, j\right) & =\left(i, \mu \pi_{T} \phi\left(i, h^{\prime}, u, h, j\right), j\right)=\left(i, h^{\prime} \pi_{s}(u) h, j\right)=\left(i, h^{\prime} \pi_{s}(v) h, j\right) \\
& =\left(i, \mu \pi_{T} \phi\left(i, h^{\prime}, v, h, j\right), j\right)=\pi_{T} \phi\left(i, h^{\prime}, v, h, j\right)
\end{aligned}
$$

and thus all the relations (21) hold in $T$. That all the other relations (22)-(25) hold in $T$ we proved in Lemma 4.1.

To complete the proof of the theorem, we show that any relation $w_{1}=w_{2}$ $\left(w_{1}, w_{2} \in(A \cup B)^{+}\right)$holding in $T$ is a consequence of the relations (21)-(25). Recall that $\sigma\left(w_{1}\right), \sigma\left(w_{2}\right) \in W \cup B$. Note that the words from $W$ represent decomposable elements of $T$, while the letters from $B$ represent indecomposable elements of $T$. Therefore, we have $\sigma\left(w_{1}\right) \in B$ if and only if $\sigma\left(w_{2}\right) \in B$. Also note that distinct letters from $B$ represents distinct elements of $T$ by (13). Thus, if $\sigma\left(w_{1}\right), \sigma\left(w_{2}\right) \in B$, then we must have $\sigma\left(w_{1}\right) \equiv \sigma\left(w_{2}\right)$, and then we have $w_{1}=\sigma\left(w_{1}\right) \equiv \sigma\left(w_{2}\right)=w_{2}$ as a consequence of the relations (22)-(25), by Lemma 4.2 .

For the remainder of this proof, we consider the case where $\sigma\left(w_{1}\right), \sigma\left(w_{1}\right) \in W$. We proceed in three steps. 
Step 1: The relation $\psi\left(w_{1}\right)=\psi\left(w_{2}\right)$ holds in S. Indeed, by Lemma 4.3 (i), we have

$$
\pi_{S} \psi\left(w_{1}\right)=\mu \pi_{T}\left(w_{1}\right)=\mu \pi_{T}\left(w_{2}\right)=\pi_{S} \psi\left(w_{2}\right)
$$

Step 2: The relation $\phi\left(i, 1, \psi\left(w_{1}\right), 1, j\right)=\phi\left(i, 1, \psi\left(w_{2}\right), 1, j\right)$ is a consequence of the relations (21). From Step 1, it follows that $\psi\left(w_{2}\right)$ can be obtained from $\psi\left(w_{1}\right)$ by applying relations from $R$. Without loss of generality, we may assume that it can be obtained by one application of one relation from $R$, say

$$
\psi\left(w_{1}\right) \equiv \alpha u \beta \text { and } \psi\left(w_{2}\right) \equiv \alpha v \beta,
$$

where $\alpha, \beta \in C^{*},(u=v) \in R$. If both $\alpha$ and $\beta$ are non-empty we can write $\alpha \equiv \alpha_{1} c\left(h_{1}, j_{1}, i_{1}, h_{1}^{\prime}\right)$ and $\beta \equiv c\left(h_{2}, j_{2}, i_{2}, h_{2}^{\prime}\right) \beta_{1}$, and then we have

$$
\begin{aligned}
\phi\left(i, 1, \psi\left(w_{1}\right), 1, j\right) & \equiv \phi\left(i, 1, \alpha_{1} c\left(h_{1}, j_{1}, i_{1}, h_{1}^{\prime}\right) u c\left(h_{2}, j_{2}, i_{2}, h_{2}^{\prime}\right) \beta_{1}, 1, j\right) \\
& \equiv \phi\left(i, 1, \alpha_{1}, h_{1}, j_{1}\right) \phi\left(i_{1}, h_{1}^{\prime}, u, h_{2}, j_{2}\right) \phi\left(i_{2}, h_{2}^{\prime}, \beta_{1}, 1, j\right) \\
& =\phi\left(i, 1, \alpha_{1}, h_{1}, j_{1}\right) \phi\left(i_{1}, h_{1}^{\prime}, v, h_{2}, j_{2}\right) \phi\left(i_{2}, h_{2}^{\prime}, \beta_{1}, 1, j\right) \\
& \equiv \phi\left(i, 1, \alpha_{1} c\left(h_{1}, j_{1}, i_{1}, h_{1}^{\prime}\right) v c\left(h_{2}, j_{2}, i_{2}, h_{2}^{\prime}\right) \beta_{1}, 1, j\right) \\
& \equiv \phi\left(i, 1, \psi\left(w_{2}\right), 1, j\right)
\end{aligned}
$$

by using (15) and (21). The case where at least one of $\alpha$ or $\beta$ is empty is treated similarly.

Step 3: The relations $w_{k}=\phi\left(i, 1, \psi\left(w_{k}\right), 1, j\right), k=1,2$, are consequences of (22)(25). To prove this, it is enough to note that, by (14) and (20), we have $\phi\left(i, 1, \psi\left(w_{k}\right), 1, j\right)=\sigma\left(w_{k}\right)$, and then apply Lemma 4.2 .

The proof of the theorem is now complete.

Corollary 4.5. Let $S$ be a semigroup, let $T=\mathcal{M}[S ; I, J ; P]$ be a Rees matrix semigroup, and let $U$ be the ideal of $S$ generated by $\left\{p_{j i} \mid j \in J, i \in I\right\}$. If $S$ is finitely presented and if all $I, J$ and $S \backslash U$ are finite, then $T$ is finitely presented as well.

Proof. If $S$ is finitely presented then so is $U$ by [18, Theorem 4.1]. As explained at the beginning of this section, $U$ can be generated (as a semigroup) by a finite set $Y=\left\{h p_{j i} h^{\prime} \mid h, h^{\prime} \in H, j \in J, i \in I\right\}$ where $1 \in H \subseteq S^{1}$. Moreover, $U$ can be defined by a finite presentation $\langle C \mid R\rangle$ in terms of $Y$. From the previous theorem, it follows that $T^{\prime}=\mathcal{M}\left[S^{1} ; I, J ; P\right]$ is finitely presented. Finally, note that $T^{\prime} \backslash T \subseteq I \times\{1\} \times J$ is finite, and hence $T$ is finitely presented by [18, Theorem 4.1$]$.

\section{Rees matrix semigroups with zero}

One common variant of the Rees matrix construction is as follows. Let $S$ be a semigroup with zero, and let $T^{\prime}=\mathcal{M}[S ; I, J ; P]$ be a Rees matrix semigroup. The set 
$I \times\{0\} \times J$ is an ideal of $T^{\prime}$. Hence it is possible to form the Rees quotient $T^{\prime} /(I \times\{0\} \times J)$ to obtain a new semigroup. This semigroup is called a Rees matrix semigroup with zero, and is denoted by $T=\mathcal{M}^{0}[S ; I, J ; P]$. It is well known that if $S=G^{0}$ is a group with a zero adjoined, and if $P$ is regular, then $T$ is a completely 0 -simple semigroup, and that all completely 0 -simple semigroups can be obtained in this way (see [15] or [5]).

Our main result of this paper remains valid for this new construction.

Main Theorem (for Rees matrix semigroups with zero). Let $S$ be a semigroup with zero, let $I$ and $J$ be index sets, let $P=\left(p_{j i}\right)_{j \in J, i \in I}$ be a $J \times I$ matrix with entries from $S$, and let $U$ be the ideal of $S$ generated by the set $\left\{p_{j i} \mid j \in J, i \in I\right\}$ of all entries of $P$. Then the Rees matrix semigroup $\mathcal{M}^{0}[S ; I, J ; P]$ is finitely generated (respectively, finitely presented) if and only if the following three conditions are satisfied:

(1) both I and $J$ are finite;

(2) $S$ is finitely generated (respectively, finitely presented); and

(3) the set $S \backslash U$ is finite.

Proof. If we let $T^{\prime}=\mathcal{M}[S ; I, J ; P]$, then we can think of $T$ as being $T^{\prime}$ with all the elements of $I \times\{0\} \times J$ being equal (and denoted by 0 ).

$(\Rightarrow)$ Assume that $T$ is finitely generated. As in Proposition 2.1 , we can prove that $I$ and $J$ are finite. Therefore the ideal $I \times\{0\} \times J$ is finite and so $T^{\prime}$ is finitely generated as well. It follows by the Main Theorem that $S$ is finitely generated and that $S \backslash U$ is finite. Moreover, if $T$ is finitely presented, then so is $T^{\prime}$ (as an ideal extension of a finite semigroup by a finitely presented semigroup). Again the Main Theorem implies that $S$ is finitely presented.

$(\Leftarrow)$ If $S$ is finitely generated and all $I, J$ and $S \backslash U$ are finite, then by the Main Theorem, $T^{\prime}$ is finitely generated. Since $T$ is a quotient of $T^{\prime}$, it follows that $T$ is finitely generated as well. Moreover, if $S$ is finitely presented then so is $T$ by the Main Theorem. Since the ideal $I \times\{0\} \times J$ is finite, it follows that $T=T^{\prime} /(I \times\{0\} \times J)$ is also finitely presented.

\section{Final remarks}

Finite presentability of Rees matrix semigroups has already been investigated in certain special cases. Thus, Howie and Ruškuc in [6] proved the converse part of the main theorem in the case where $S$ is a monoid and $P$ contains at least one invertible entry. Also, an immediate application of the Reidemeister-Schreier type rewriting technique developed in [1] proves the direct part of the Main Theorem in the case of completely (0-)simple semigroups. Finite generation and ranks of Rees matrix semigroups (in the completely (0-)simple case) have been considered in [4] and [17]. 
This paper is a part of wider research into finite presentability (and other finiteness conditions) of various semigroup constructions; see [3], [6], [16], [18]. A common feature in all these results is that of a rewriting mapping (mappings $\psi$ and $\phi$ in Sections 3 and 4). It is interesting to note that, unlike in other constructions considered so far, no rewriting mapping defined in this paper is a homomorphism. This is because, in general, $S$ is neither a subsemigroup nor a homomorphic image of $T=\mathcal{M}[S ; I, J ; P]$.

\section{REFERENCES}

1. C. M. Campbell, E. F. Robertson, N. Ruškuc and R. M. Thomas, ReidemeisterSchreier type rewriting for semigroups, Semigroup Forum 51 (1995), 47-62.

2. C. M. Campbell, E. F. Robertson, N. Ruškuc and R. M. Thomas, On subsemigroups of finitely presented semigroups, J. Algebra 180 (1996), 1-21.

3. C. M. Campeell, E. F. Robertson, N. Ruškuc and R. M. Thomas, Presentations for subsemigroups - applications to ideals of semigroups, J. Pure Appl. Algebra, to appear.

4. G. M. S. Gomes and J. M. HowIE, On the ranks of certain finite semigroups of transformations, Math. Proc. Cambridge Philos. Soc. 101 (1987), 395-403.

5. J. M. HowIE, Fundamentals of Semigroup Theory (Clarendon Press, Oxford, 1995).

6. J. M. HowIE and N. RuŠKuc, Constructions and presentations for monoids, Comm. Algebra 22 (1994), 6209-6224.

7. A. JURA, Determining ideals of given finite index in a finitely presented semigroup, Demonstratio Math. 11 (1978), 813-827.

8. M. V. Lawson, Abundant Rees matrix semigroups, J. Austral. Math. Soc. 42 (1987), 132-142.

9. M. V. Lawson, Rees matrix semigroups, Proc. Edinburgh Math. Soc. 33 (1990), 23-37.

10. D. B. MCAlister, Regular Rees matrix semigroups and regular Dubreil-Jacotin semigroups, J. Austral. Math. Soc. 31 (1981), 325-336.

11. D. B. MCALister, Quasi-ideal embeddings and Rees matrix covers for regular semigroups, J. Algebra 152 (1992), 166-183.

12. J. Meakin, Fundamental regular semigroups and the Rees construction, Quart. J. Math. Oxford (2) 36 (1985), 91-103.

13. J. MEAKIN, The Rees construction in regular semigroups, in Semigroups (Colloquia Mathematica Societatis János Bolyai Vol. 39, North-Holland, Amsterdam, 1985), 115-155.

14. F. Pastijn and M. Petrich, Rees matrix semigroups over inverse semigroups, Proc. Royal Soc. Edinburgh 102A (1986), 61-90.

15. D. Rees, On semi-groups, Proc. Cambridge Philos. Soc. 36 (1940), 387-400.

16. E. F. Robertson, N. Ruškuc and J. WIEGold, Generators and relations of direct products of semigroups, Trans. Amer. Math. Soc. 350 (1998), 2665-2685.

17. N. Ruškuc, On the rank of completely 0-simple semigroups, Math. Proc. Cambridge Philos. Soc. 116 (1994), 325-338. 
GENERATORS AND RELATIONS OF REES MATRIX SEMIGROUPS 495

18. N. Ruškuc, On large subsemigroups and finiteness conditions of semigroups, Proc. London Math. Soc. 76 (1998), 383-405.

19. A. SuschKewitsCh, Über die endlichen Gruppen ohne das Gesetz der eindeutigen Umkehrbarkeit, Math. Ann. 99 (1928), 30-40.

Mathematical INSTITUTe

UNIVERSITY OF ST ANDREWS

ST ANDREWS KY16 9SS

SCOTLAND

E-mail address: ayik@dcs.st-and.ac.uk,nik@dcs.st-and.ac.uk 